\title{
Photothermal Conversion and Electrical Characteristics of Laser-Induced Graphite Carbon
}

\author{
Zhang Qiurui, Jia GuOzhi*, Zhao ZeiJia, \\ ZhOU YaOYAO AND Li LingXia
}

School of Science, Tianjin Chengjian University, Tianjin 300384, China

Received: 12.11.2019 \& Accepted: 24.06.2020

Doi: 10.12693/APhysPolA.138.399

*e-mail: jiaguozhi@tcu.edu.cn

\begin{abstract}
The graphitic carbon has been widely investigated due to excellent optical and electrical performance. The graphitic carbon structure is prepared by a laser-induced technology. The microscopic morphology of the porous structures is analyzed through optical microscopy and scanning electron microscopy. It is confirmed that graphitic carbon is formed on the basis of the Raman spectrum analysis. The effect of the laser power and scanning rate on the photothermal conversion and electrical characteristics of the graphitic carbon film are investigated in detail. The electrical conductivity increases with the growing laser power and action time. The ability of photothermal conversion increases with the growing scanning rates, and reaches the highest temperature under the laser scanning rate of $5 \mathrm{~cm} / \mathrm{s}$ at the fixed laser power of $4.5 \mathrm{~W}$. Finally, the physics mechanism is discussed in depth
\end{abstract}

topics: laser-induced, graphitic carbon, photothermal conversion, electrical characteristics

\section{Introduction}

The two-dimensional material graphene has widely been investigated in supercapacitors [1], biotherapy [2], and sensors [3] due to its unique optical and electrical properties. A variety of physical and chemical methods have been used to prepare graphene, such as mechanical exfoliation [4], chemical vapor deposition [5], and Hummers method [6]. Despite tremendous advances in the preparation of graphene, a simple and low-cost method is still desired for its commercialization to substitute the complicated synthetic route in practical application. Graphene or graphitic carbon can be rapidly prepared by a laser direct writing (LDW) technology based on the photothermal or photochemical reduction principle [7]. As for wearable electronics [8] and flexible energy storage devices $[1,9]$, the patterning $[9,10]$ and in-situ fabricating graphene [11] nanomaterials are further desired for future commercial applications.

LDW can generate micro/nano-structures and prepare nanomaterials via a high temperature ablative and/or photochemical process [12, 13]. Laserinduced graphene is a multi-process synergistic reaction to prepare nanomaterials by destroying and recombining a chemical bond under the influence of high photon energy $[11,14,15]$. The parameters of a laser, such as the laser power and scanning rate, have a very important effect on the electrical performance of materials. In addition, it is essential to study the photothermal conversion of the LDW microstructure for the potential photo-to-heat and thermoelectricity application $[16,17]$.
Here, we use laser-induced technology to prepare porous graphitic carbon and further study its photothermal conversion ability and electronic characteristics. The laser-induced graphitic carbon has an excellent photothermal conversion capability when it is irradiated by using a $808 \mathrm{~nm}$ laser with a power $0.6 \mathrm{~W}$, and its line resistance can reach about $30 \mathrm{ohms} / \mathrm{cm}$. The physics mechanism is further discussed in detail.

\section{Experiments}

In this experiment, the commercial polyimide (PI) film with the thickness of $0.08 \mathrm{~mm}$ is obtained from Tianjin Jiayin. The PI film is cut into a square having a surface of $5 \times 5 \mathrm{~cm}^{2}$, and is carefully treated in alcohol solution. The PI film is then pasted onto a glossy photo paper and the bubbles are removed to ensure the surface flatness. The designed graphics can be formed by the acting PI film under ambient conditions through a $450 \mathrm{~nm}$ wavelength laser direct writing system (Tianjin Jiayin). The maximum power of the laser device is $5.5 \mathrm{~W}$ and the diameter of the focused laser spot is about $100 \mu \mathrm{m}$. In this system, the laser spot is precisely adjusted in the $x$ and $y$ directions by a scanning system (the working area is $20 \times 20 \mathrm{~cm}^{2}$ ). The laser radiates directly on the PI film, according to the predefined patterns and parameters, as shown in Fig. 1a. All of the laser experiments are performed under ambient conditions.

The surface morphology of laser-induced graphitic carbon is analyzed by optical microscopy (DMM-330C, Gold phase optical microscope, 
(a)
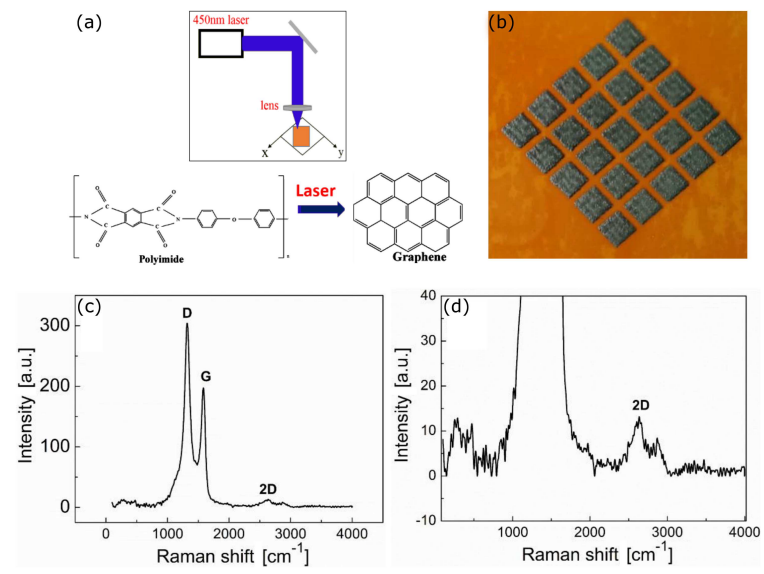

Fig. 1. (a) Laser-induced graphitic carbon process schematic. (b) The square matrix sample of graphitic carbon. (c) Raman spectra of the carbonized area (sample: laser power: $140 \mathrm{~mW}$, scanning speed: $1 \mathrm{~mm} / \mathrm{s}$ ). (d) Expansion figure of the $2 \mathrm{D}$ peak from $(\mathrm{c})$.

Shanghai) and scanning electron microscopy (SEM Hitachi SU-8020, Hitachi Company, Tokyo, Japan). Raman spectra are collected via Micro Raman spectroscopy system (Renishaw) equipped with a laser power of $5 \mathrm{~mW}$ at a $514 \mathrm{~nm}$ laser excitation. For measuring the photothermal conversion performance of graphitic carbon, a $808 \mathrm{~nm}$ NIR laser beam is used. The resistance is measured using a standard multimeter (UNI-T UT58B).

\section{Results and discussion}

Raman spectroscopy is used to distinguish chemical compounds and molecular structures of the PI material after laser treatment. As shown in Fig. 1c, the Raman peaks of $\mathrm{D}, \mathrm{G}$, and $2 \mathrm{D}$ emerging at $1325 \mathrm{~cm}^{-1}, 1597 \mathrm{~cm}^{-1}$ and $2639 \mathrm{~cm}^{-1}$ are detected under excitation of laser energy, respectively. The $\mathrm{D}$ peak is induced by defects or bent $s p^{2}$-carbon bonds in the graphitic lattice, the $G$ and $2 \mathrm{D}$ peaks originate from a second-order zone-boundary phonon [18, 19]. The defects can be quantified by analyzing the ratio of the peak intensity $I_{\mathrm{D}} / I_{\mathrm{G}}[18,20]$. A number of defects can be formed in the laser-induced graphitic carbon on polyimide materials due to breaking the translational symmetry which indicates the formation of carbonation and graphitic carbon. It can be concluded that the semiconductor laser can convert PI into graphitic carbon or carbon hybridization $[11,12]$. The in-plane crystallite sizes of the laser-induced graphitic carbon can be determined by an empirical expression derived from typical characteristic peaks of Raman spectra [21, 22]. A relative report on the grain size of graphitic carbon has been discussed as [21]:

$$
L_{a}=2 \times 10^{-10} \lambda^{4}\left(\frac{I_{\mathrm{D}}}{I_{\mathrm{G}}}\right)^{-1},
$$
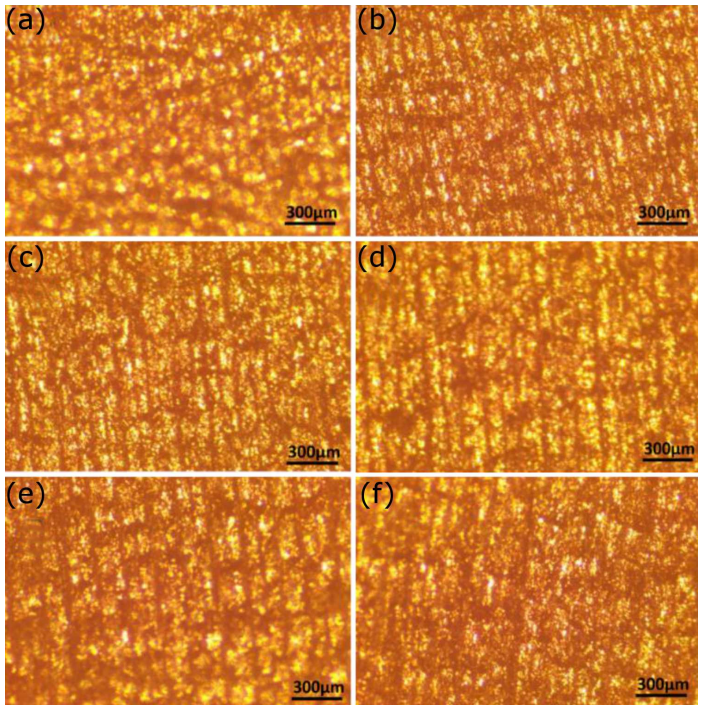

Fig. 2. The graphitic carbon surface is analyzed by an optical microscope, PI film is treated by the laser power of $4.5 \mathrm{~W}$ and different laser scan rates: (a) $15 \mathrm{~cm} / \mathrm{s}$, (b) $7.5 \mathrm{~cm} / \mathrm{s}$, (c) $5 \mathrm{~cm} / \mathrm{s}$, (d) $3.75 \mathrm{~cm} / \mathrm{s}$, (e) $3 \mathrm{~cm} / \mathrm{s}$, (f) $2.5 \mathrm{~cm} / \mathrm{s}$.

where $L_{a}$ represents the size of the grain, $\lambda$ is the wavelength of the Raman laser excitation $(514 \mathrm{~nm})$ and the $I_{\mathrm{D}} / I_{\mathrm{G}}$ denotes the $\mathrm{D}$ and $\mathrm{G}$ area integrate peaks ratio from the Raman feature. The size of the graphitic carbon nanocrystal is calculated as $45.9 \mathrm{~nm}$, according to the formula.

The action time of the laser plays an important role in the electrical, optical, and thermal characteristics of the thin film. The surface structure of laser treatment areas at different laser scanning rates at the fixed power $(4.5 \mathrm{~W})$ is observed using an optical microscope, as shown in Fig. 2. It can clearly be seen that the PI surface performs clear patterns when the laser scanning rates are $7.5 \mathrm{~cm} / \mathrm{s}$ and $5 \mathrm{~cm} / \mathrm{s}$, while the boundary distinction is blurred for slow scan rates $(3.75 \mathrm{~cm} / \mathrm{s}, 3 \mathrm{~cm} / \mathrm{s}$ and $2.5 \mathrm{~cm} / \mathrm{s})$ and a fast scan rate $(15 \mathrm{~cm} / \mathrm{s})$. The laser scanning rate determines the laser action time which directly affects the conversion degree of graphitic carbon. The slow scanning speed may also lead to a large thermal expansion, and the overlap observation between the strips is obvious. The slow scanning speed may also result in discontinuous transformation of graphitic carbon due to the appearance and transformation of some regions. The high energy density of the laser is an important factor to ensure that the PI film reaches the conversion heating temperature. The degree of carbonization depends on the duration of the laser action and on the laser power. In addition, the degree and depth of carbonization will increase with the laser power growth.

Figure 3a shows the surface morphology characterization of the carbonized PI surface analyzed by the scanning electron microscopy with the laser power fixed at $1 \mathrm{~W}$ and the scan rate of $0.2 \mathrm{~cm} / \mathrm{s}$. 
A foamy-like surface is generated on the PI surface with the line width of $\approx 100 \mu \mathrm{m}$ due to a rapid liberation of gaseous products. Figure $3 \mathrm{~b}$ shows that carbonized films exhibit a porous network structure due to a large amount of gas produced during the process of laser treatment. The pore size distribution range of porous nanostructures is 2 to $10 \mu \mathrm{m}$. It is worth noting that unique porous structures render a high surface area to enhance their light absorption performance. The formation of these honeycomb-like carbonized structures is ascribed to the synergistic effects of the photo heat and optical chemistry during the irradiation process of the short wavelength semiconductor laser [14].

The effect of a scanning rate on photothermal conversion performance [23] is studied in depth. The irradiation light source is an $808 \mathrm{~nm}$ wavelength near-infrared laser with the power of $0.6 \mathrm{~W}$. Figure $4 \mathrm{a}$ shows the photothermal conversion performance of the samples on which the laser acted with the fixed power of $4.5 \mathrm{~W}$, with different scanning rates from 0 to $5 \mathrm{~cm} / \mathrm{s}$. The temperature of carbonized film reaches $114.2{ }^{\circ} \mathrm{C}$ for the sample with the $5 \mathrm{~cm} / \mathrm{s}$ scanning rate at the time below $150 \mathrm{~s}$ while a blank test shows that the temperature change of the PI film is only $20^{\circ} \mathrm{C}$ in the same conditions. The excellent photothermal conversion ability can be ascribed to the porous surface morphology and carbonized characterization. It may be concluded that the carbonized film can rapidly convert the $808 \mathrm{~nm}$ laser energy into heat which can be attributed to the strong NIR absorption and good thermal insulation function. Figure $4 \mathrm{~b}$ shows that the carbonized PI surface temperature is predominantly maintained at around $110^{\circ} \mathrm{C}$ and the open measuring environment can lead to a small difference of temperature for the samples with the scanning rate from $3 \mathrm{~cm} / \mathrm{s}$ to $7.5 \mathrm{~cm} / \mathrm{s}$. As for samples with a laser scanning rate of $2.5 \mathrm{~cm} / \mathrm{s}$, the low saturation temperature may result from longer laser irradiation due to the carbide on the surface of the sample leaving the PI film. Rapid heating and high balance temperature will be useful for the fields that require fast temperature switching and micro heating device.

As shown in Fig. 4c, temperature variation of carbonized film increases and then slightly goes down with the growth of the laser scanning rate under the $808 \mathrm{~nm}$ laser light illumination, which can be ascribed to two key factors. The amount of the graphitic carbon production plays an important role during the process of photothermal conversion. The amount of the forming graphitic carbon increases with decreasing of the laser scanning rate. In addition, a large laser scanning rate can lead to removing a part of the carbonized film. It is necessary to point out that a faster heat loss can slightly influence the temperature variation at open environment. The heating rate is mainly changed from $5^{\circ} \mathrm{C} / \mathrm{s}$ to $6^{\circ} \mathrm{C} / \mathrm{s}$ with the laser scanning rate increasing from $3 \mathrm{~cm} / \mathrm{s}$ to $15 \mathrm{~cm} / \mathrm{s}$. The heat loss

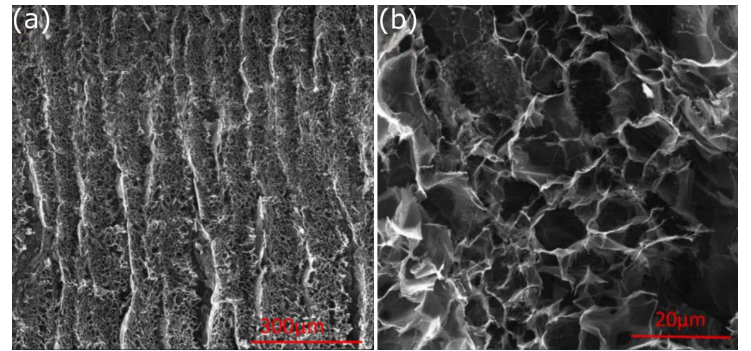

Fig. 3. The surface morphology of the porous graphitic carbon is characterized by a scanning electron microscope.
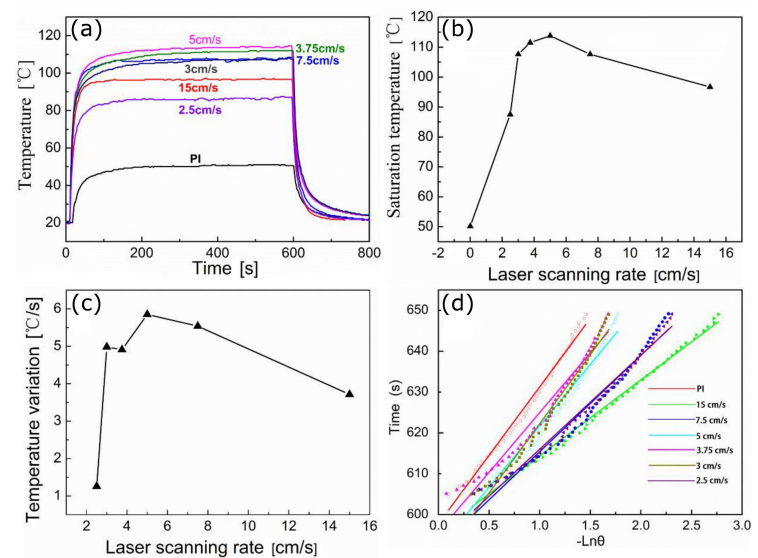

Fig. 4. (a) The photothermal conversion scheme of graphitic carbon and carbide hybridization film irradiated by an $808 \mathrm{~nm}$ near-infrared laser with different speed; the laser is turned off after irradiation for $10 \mathrm{~min}$. (b) The saturation temperature via different laser scanning rates. (c) The speed of film reaches its maximum temperature via the laser scanning rate. (d) The heat transfer time constants are calculated by fitting.

of environmental exchange is larger due to a larger carbonized pore size when the laser scanning rate is $2.5 \mathrm{~cm} / \mathrm{s}$. The thermal equilibrium time constant for the heat transfer time can be obtained using temperature versus time data recorded during the cooling of the solution (Fig. 4d) based on the energy balance equation $[24,25]$. Figure $4 d$ shows the time constant of the heat transfer time which is determined as the negative reciprocal slope of $\ln (\theta)$ and $t$ with the temperature and time data recorded during the solution cooling. It can be seen that the transfer time $t$ of laser-induced graphitic carbon is shorter than that of polyimide which may be due to the fact that the absorption of nearinfrared light by graphitic carbon is higher than that of polyimide. In addition, the porous graphitic carbon structure may lead to the multiple diffuse scattering of light in the graphitic carbon film to be fully absorbed. It is consistent with the result in Fig. 4a, further proving that laser-induced graphitic carbon has more advantages than polyimide's good thermal performance. 

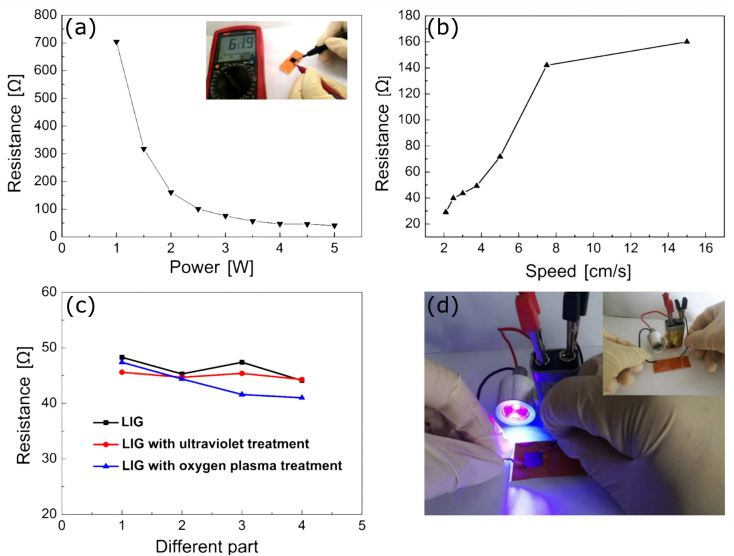

Fig. 5. (a) The variation of resistance with the laser power increase at the fixed laser scanning rate. (b) The variation of resistance with a different laser scanning rate at the same laser power. (c) The variation of resistance after treating by oxygen plasma and ultraviolet light. (d) The laser-induced graphitic carbon can make the bulb emit light, but polyimide cannot.

In order to accurately measure the resistance between two points, an electrical test device is constructed to guarantee the same contact force, distance and other conditions for each measurement. The laser-induced graphitic carbon has a compact network of graphitic carbon flakes, thus the measurement direction does not affect the resistance value (other measurement conditions are the same). The resistance can only be measured in the direction of the laser movement in the experimental measurement process. Figure 5a shows that the resistance value per unit length decreases as the laser power increases. As the power increases, the degree of hybridization of graphitic carbon and carbon increases leading to the line resistance decrease. Figure 5b shows that polyimide is irradiated through changing the scanning rate at the same laser power $(4.5 \mathrm{~W})$. The line resistance of each sample is measured (measurement distance is $1 \mathrm{~cm}$ ), and it is found that the conductivity increased as the laser scan rate decreased. This can be ascribed to the yield of graphitic carbon increases as the rate decreases, which can lead to more electronics transport channels formed [26]. The high laser scanning rate can lead to producing various cracks and the graphitic carbon falling off from the film due to laser treatment. Figure $5 \mathrm{c}$ shows that the resistance of the laser-induced graphitic carbon has not obviously changed after treating by oxygen plasma and ultraviolet light, indicating that the LIG film exhibits excellent stability. The graphitic carbon film shows good electronic circuit for the conductivity application, as shown in Fig. 5d.

The result of the resistance measurement shown in Fig. $5 \mathrm{a}-\mathrm{b}$ is the resistance measured by a twopoint method. Relative to the surface resistance, a four-point method is much better than a two-point method. Understanding the electrical characteristics of the laser action is partly the focus of this paper. There are many factors that affect the measurement of resistance by the two-point method, for example contact resistance. A fixed device is used to measure the resistance to avoid human influence as much as possible, which can ensure the distance between probes and the working pressure of probes so as to secure the accuracy of measurement. In addition, the resistance is an anisotropic relative to the direction of the line. The resistance along the laser scanning direction is smaller than that perpendicular to the laser scanning direction. There are two main reasons for that. One is that there are very small intervals perpendicular to the laser scanning direction. These intervals may not be enough depth-affected by the laser, resulting in greater resistance. The other is that these intervals may only be burned by the laser and they are not involved in the process of laser photo reduction.

The mechanism of conversion of polyimide into graphitic carbon is discussed by considering the photothermal $[26,27]$ and photochemical $[28,29]$ processes. Firstly, the photothermal effect may play an important role in the laser-induced graphitic carbon process. An incandescent state is presented in the laser-induced graphitic carbon process which can lead to the temperature of laser acting on the material reach around $1700^{\circ} \mathrm{C}$ [11]. The heat causes lattice vibrations [30] and destroys the $\mathrm{C}-\mathrm{O}, \mathrm{C}=\mathrm{O}$ and $\mathrm{N}-\mathrm{H}$ bonds in the imide ring (-CO-NH-CO-) [31]. Most of the oxygen and nitrogen are removed during the process of the porous structure forming due to producing gas. In addition, the photochemical process may be another key factor because it generally occurs in a shorter wavelength $(450 \mathrm{~nm})$ ultraviolet laser [32, 33]. In the laser-induced graphitic carbon forming process, the amount of graphitic carbon can increase with the increase of the laser power and action time which can increase the ratio of graphitic carbon formed on the surface of the polyimide. Note that the formation of a graphitic carbon network leads to a further increase of the conductivity. As for the photothermal conversion, the pore structure plays a key role in excellent photo absorbtion through the process of many time diffractions. Therefore, the number, shape and size of the pores have a great influence on the temperature increase and heat energy storage.

\section{Conclusions}

Graphitic carbon can be prepared by a one-step laser-induced technique. The semiconductor laser can convert polyimide into graphitic carbon or carbon hybridization based on analyzing the Raman spectrum. It is found that the electrical conductivity of porous graphitic carbon grows with the laser power increasing and the scanning rate decreasing, and the optimized resistance value $(30 \mathrm{ohms} / \mathrm{cm})$ 
can be obtained by changing the laser parameters. The highest photothermal conversion temperature of the thin film can reach $114.6{ }^{\circ} \mathrm{C}$ under the action of the $808 \mathrm{~nm}$ laser with the power of $0.6 \mathrm{~W}$. The test result further shows that the laser-induced graphitic carbon exhibits an excellent thermal conversion capability and favorable electrical conductivity for the electrical and photothermal application.

\section{Acknowledgments}

This work has been supported by the National Natural Science Foundation of China (11674240) and the key project of the Natural Science Foundation of Tianjin (19JCZDJC38600).

\section{References}

[1] M.F. El-Kady, R.B Kaner, Nat. Commun. 4, 1475 (2013).

[2] C.H. Fu, L. Qiang, Q.H. Liang, X. Chen, L.L. Li, H.Y. Liu, L.F. Tan, T.L. Liu, X.L. Ren, X.W. Meng, RSC Adv. 5, 46158 (2015).

[3] S.D. Luo, P.T.Hoang, T. Liu, Carbon 96, 522 (2016).

[4] K.S. Novoselov, A.K. Geim, S.V. Morozov, D. Jiang, Y. Zhang, S.V. Dubonos, I.V. Grigorieva, A.A. Firsov, Science 306, 666 (2004).

[5] J. Ryu, Y. Kim, D. Won et al. ACS Nano 8, 950 (2014).

[6] V.M. Boychuk, V.O. Kotsyubynsky, K.V. Bandura, I.P. Yaremiy, S.V. Fedorchenko, J. Nanosci. Nanotechnol. 19, 7320 (2019).

[7] F. Mahmood, C. Zhang, Y.C. Xie, D. Stalla, J. Lin, C.X. Wan, $R S C A d v .9$, 22713 (2019).

[8] C. Mattmann, F. Clemens, G. Troester, Sensors 8, 3719 (2008).

[9] H. Tian, H.-Y. Chen, T.-L. Ren, C. Li, Q.-T. Xue, M.A. Mohammad, C. Wu, Y. Yang, H.S.P. Wong, Nano Lett. 14, 3214 (2014).

[10] Y. Xie, L. Wen, J. Zhang, T. Zhou, Mater. Des. 141, 159 (2018).

[11] J. Lin, Z. Peng, Y. Liu, F. Ruiz-Zepeda, R. Ye, E.L.G. Samuel, M.J. Yacaman, B.I. Yakobson, J.M. Tour, Nat. Commun. 5, 5714 (2014).

[12] J. Bin In, B. Hsia, J.-H. Yoo, S. Hyun, C. Carraro, R. Maboudian, C.P. Grigoropoulos, Carbon 83, 144 (2015).

[13] C.G. Zhu, D.M. Zhao, K.D. Wang, X. Dong, W.Q. Duan, F.C. Wang, M. Gao, G. Zhang, J. Mater. Sci. 54, 4192 (2019).
[14] J. Zhu, X. Guo, H. Wang, W. Song, J. Mater. Sci. 53, 12413 (2018).

[15] W. Song, J. Zhu, B. Gan, S. Zhao, H. Wang, C. Li, J. Wang, Small 14, 1702249 (2018).

[16] Y. Zhang, G. Jia, P. Wang, AIP Adv. 7, 045210 (2017).

[17] Z. Zhang, M. Song, J. Hao, K. Wu, C. Li, C. $\mathrm{Hu}$, Carbon 127, 287 (2018).

[18] R. Beams, L.G. Cancado, L. Novotny, J. Phys. Condens. Matter 27, 083002 (2015).

[19] J.S. Park, A. Reina, R. Saito, J. Kong, G. Dresselhaus, M.S. Dresselhaus, Carbon 47, 1303 (2009).

[20] A.C. Ferrari, J.C. Meyer, V. Scardaci et al. Phys. Rev. Lett. 97, 187401 (2006).

[21] L.G. Cancado, K. Takai, T. Enoki, M. Endo, Y.A. Kim, H. Mizusaki, A. Jorio, L.N. Coelho, R. MagalhaesPaniago, M.A. Pimenta, Appl. Phys. Lett. 88, 163106 (2006).

[22] A.M. Dimiev, G. Ceriotti, N. Behabtu, D. Zakhidov, M. Pasquali, R. Saito, J.M. Tour, ACS Nano 7, 2773 (2013).

[23] G. Jia, P. Wang, Y. Zhang, K. Chang, Sci. Rep. 6, 25884 (2016).

[24] G.Z. Jia, W.K. Lou, F. Cheng, X.L. Wang, J.H. Yao, N. Dai, H.Q. Lin, K. Chang, Nano Res. 8, 1443 (2015).

[25] Y. Zhang, G. Jia, P. Wang, Q. Zhang, X. Wei, E. Dong, J. Yao, Superlattice Microstruct. 105, 22 (2017).

[26] A.O. Diachenko, T.V. Panchenko, Acta Phys. Pol. A 133, 936 (2018).

[27] Z. Er, Acta Phys. Pol. A 132, 1025 (2017).

[28] Z. Wang, F. Liu, J. Li, C. He, X. Peng, Z. Huang, P. Fang, Acta Phys. Pol. A 132, 1523 (2017).

[29] F. Azzouz, S. Kaci, I. Bozetine, A. Keffous, M. Trari, S. Belhousse, S. Aissiou-Bouanik, Acta Phys. Pol. A 132, 479 (2017).

[30] A. Iller, W. Jantsch, J. Marks, B. Pastuszka, R. Diduszko, Acta Phys. Pol. A 94, 336 (1998).

[31] D.K. Cheng, H. Wang, B. Liu, S. Wang, Y. Li, Y.S. Xia, C.X. Xiong, J. Appl. Polym. Sci. 136, 7 (2019).

[32] V. Vinciunaite, A. Grigonis, A. Medvid, R. Zabels, Acta Phys. Pol. A 123, 874 (2013).

[33] W.A. Farooq, M.R. Baig, A. Fatehmulla, M.S. Al-Salhi, S.S. Al-Ghamdi, F. Yakuphanoglu, Acta Phys. Pol. A 123, 106 (2013). 